\title{
Tele-health in the era of COVID-19 pandemic: Pros and
}

\section{cons}

\author{
MD. Abu Bashar ${ }^{*}$, Nazia Begam ${ }^{2}$ and Sachin Gupta \\ ${ }^{1}$ Institute of Medical Sciences, BHU, Varanasi, India \\ ${ }^{2}$ ECHS Hopital, Ambala, Haryana, India \\ ${ }^{3}$ ESI Poly Clinic, Baddi, HP, India
}

The Covid-19 pandemic has radically altered how we provide care to our patients, propelling us into the world of virtual healthcare delivery at a dizzying pace. Tele-Health and its digital accoutrements offer an exciting new avenue for patient care. But the transformation has not been perfect, and both patients and providers are learning to navigate a new terrain of care provision, often without clear guidelines or support.

This new frontier has brought a virtual twist to the classic "patient encounter" stories we share-Tele-Health providers recount blundered exchanges, gaffs, and missed and misinterpreted nonverbal cues. These stories bring a new flavour to our encounters with patients-they represent the most human interactions, in a time when humanity has been forced to interact in all kinds of new and uncomfortable ways. We are growing and learning with our patients, gradually coalescing around a shared set of practices and beliefs to anchor our virtual community.

But there are also stories about virtual care which are not so funny; sadly, many of these come from patients. Indifferent-seeming physicians who don't make eye contact, or who are distracted by their mobile phones. Concerns about excessively short visit times, or what the lack of a physical exam means. Worries about privacy, confidentiality, and security. Language barriers. Hearing, vision, and other adaptive needs that aren't being met. These encounters are less humorous when told from the perspective of an anxious or unwell patient, and they reflect a darker side of this new world that must be addressed if we expect our patients to continue present to care Digital professionalism - the adaptation of professional behaviour, codes of conduct, and social norms into virtual spaces-is acknowledged by the medical community as an important component of modern healthcare delivery, and is one area in which we are building tools to ensure high-quality care for patients [1,2]. In the United States, the Federation of State Medical Boards offers policy guidelines for appropriate use of the internet, social media, and digital social networking in medical practice; the American Medical Association addresses many elements of digital professionalism-including individual behaviour, confidentiality and consent, prescribing, and financial disclosures-in its statement on ethical practice in telemedicine [3-5]. In medical education, there are calls to adapt professionalism milestones for trainees to digital domains.

A related but less recognized concept is that of digital empathy or digital compassion. In their article "The Emerging Issue of Digital Empathy", Christopher Terry and Jeff Cains define digital empathy as "traditional empathic characteristics such as concern and caring for others expressed through computer-mediated communications" [6].
Similarly, David Wiljer identifies "digital compassion" as the experience that an individual has on recognizing and wanting to alleviate the suffering or perceived unmet needs of another in digital space [7]. These conception go beyond recommendations on how to draft a professional email or curate a social media account; they challenge us to re-evaluate how we connect with our patients.

Both authors point to the rapid expansion and adoption of digital technology as a disruptor of patterns in human interaction; this disruption can impact people's ability to connect, empathize, and care for one another in detrimental ways-a "digital dis-inhibition".

Digital empathy and compassion offer helpful constructs in thinking about virtual healthcare delivery, counteracting the tendencies of digital dis-inhibition and reinforcing caring social relationships between patients and providers. A strong foundation in digital empathy can help us acknowledge challenging moments in virtual encounters and partner with our patients to work through them. But more research is needed on how to conceptualize these constructs and operationalize them in virtual practice. Similarly, the impact of digital dis-inhibition on the patient-provider relationship must be better understood if we are to adequately build and scale virtual health services.

The idea of virtual care as "the next best thing" in healthcare delivery is deeply alluring. It conjures a world of easy access to highquality care regardless of demographics or social determinants-a great democratizer. But a virtual healthcare system isn't a panacea. The distance created by virtual health environments tests the limits of our ability to be caring, compassionate providers; this threatens our identity as physicians and places our already vulnerable patients at risk. To address this, we must challenge the assumption that all the work we've done to build ourselves as caring, compassionate providers will simply translate as it is virtualized.

\section{References}

1. Ellaway R, Coral J, Topps D, Topps M (2015) Exploring digital professionalism. Medical Teacher 37: 844-849.

2. American Academy of Medical Colleges. AAMC. Viewpoint - Digital Professionalism. https://www.aamc.org/professional-development/affinity-groups/gir/viewpoint-digitalprofessionalism. Accessed 12/20/2020.

*Correspondence to: MD. Abu Bashar, Institute of Medical Sciences, BHU, Varanasi, India, E-mail: dr.bashartheultimate86@gmail.com

Received: January 19, 2021; Accepted: February 10, 2021; Published: February 15,2021 
3. Federation of State Medical Board guidelines on social media and electronic communications. www.fsmb.org/pdf/pub-social-media-guidelines.pdf. Accessed $12 / 19 / 2020$

4. American Medical Association. Ethical practice in telemedicine. https://www.amaassn.org/delivering-care/ethics/ethical-practice-telemedicine. Accessed 12/18/2020.
5. Terry C, Cain J (2016) The emerging issue of digital empathy. Am J Pharm Educ 80: 58.

6. Wiljer D, Charow R, Costin H, Sequeira L, Anderson M, et al. (2019) Defining compassion in the digital health age: protocol for a scoping review. BMJ Open 9: $\mathrm{e} 026338$.

7. Suler J (2004) The online disinhibition effect. Cyberpsychol Behav 7: 321-326.

Copyright: $\mathbb{C} 2021$ Abu Bashar MD. This is an open-access article distributed under the terms of the Creative Commons Attribution License, which permits unrestricted use, distribution, and reproduction in any medium, provided the original author and source are credited. 